\title{
Kaleidoscopical configurations in $G$-spaces
}

\author{
Taras Banakh
}

Faculty of Mechanics and Mathematics

Lviv University, Universytetska 1, Lviv 79000, Ukraine

t.o.banakh@gmail.com

\section{Oleksandr Petrenko}

Faculty of Cybernetics

Kyiv University, Volodymyrska 64, Kyiv 01033, Ukraine

opetrenko72@gmail.com

Igor Protasov

Faculty of Cybernetics

Kyiv University, Volodymyrska 64, Kyiv 01033, Ukraine

i.v.protasov@gmail.com

Sergiy Slobodianiuk

Faculty of Mechanics and Mathematics

Kyiv University, Volodymyrska 64, Kyiv 01033, Ukraine

slobodianiuk@yandex.ru

Submitted: Jan 27, 2011; Accepted: Dec 13, 2011; Published: Jan 6, 2012

Mathematics Subject Classifications: 05B45, 05C15, 05E15, 05E18, 20K01

\begin{abstract}
Let $G$ be a group and $X$ be a $G$-space with the action $G \times X \rightarrow X,(g, x) \mapsto g x$. A subset $F$ of $X$ is called a kaleidoscopical configuration if there exists a coloring $\chi: X \rightarrow C$ such that the restriction of $\chi$ on each subset $g F, g \in G$, is a bijection. We present a construction (called the splitting construction) of kaleidoscopical configurations in an arbitrary $G$-space, reduce the problem of characterization of kaleidoscopical configurations in a finite Abelian group $G$ to a factorization of $G$ into two subsets, and describe all kaleidoscopical configurations in isometrically homogeneous ultrametric spaces with finite distance scale. Also we construct $2^{\mathfrak{c}}$ (unsplittable) kaleidoscopical configurations of cardinality $\mathfrak{c}$ in the Euclidean space $\mathbb{R}^{n}$.
\end{abstract}




\section{Introduction}

Let $X$ be a set and $\mathfrak{F}$ be a family of subsets of $X$ (the pair $(X, \mathfrak{F})$ is called a hypergraph). Following [4], we say that a coloring $\chi: X \rightarrow \kappa$ of $X$ (i.e. a mapping of $X$ onto a cardinal $\kappa)$ is

- $\mathfrak{F}$-surjective if the restriction $\left.\chi\right|_{F}$ is surjective for all $F \in \mathfrak{F}$;

- $\mathfrak{F}$-injective if $\left.\chi\right|_{F}$ is injective for all $F \in \mathfrak{F}$;

- $\mathfrak{F}$-bijective or $\mathfrak{F}$-kaleidoscopical if $\left.\chi\right|_{F}$ is bijective for all $F \in \mathfrak{F}$.

A hypergraph $(X, \mathfrak{F})$ is called kaleidoscopical if there exists an $\mathfrak{F}$-kaleidoscopical coloring $\chi: X \rightarrow \kappa$. The adjective "kaleidoscopical" appeared in definition [5] of an s-regular graph $\Gamma(V, E)$ (each vertex $v \in V$ has degree $s$ ) admitting a vertex $(s+1)$-colloring such that each unit ball $B(v, 1)=\{u \in V: d(u, v)=1\}$ has the vertices of all colors $(d$ is a path metric on $V$ ). These graphs can be considered as a graph counterpart of Hamming codes [6].

We shall consider hypergraphs related to a $G$-space. Let $G$ be a group. A $G$-space is a set $X$ endowed with an action $G \times X \rightarrow X,(g, x) \mapsto g x$. All $G$-spaces are suppose to be transitive (for any $x, y \in X$ there exists $g \in G$ such that $g x=y$ ). For a subset $A \subseteq X$, we put $G[A]=\{g A: g \in G\}$.

A subset $A \subseteq X$ is called a kaleidoscopical configuration if the hypergraph $(X, G[A])$ is kaleidoscopical (in words, if there exists a coloring $\chi: X \rightarrow|A|$ such that $\left.\chi\right|_{g A}$ is bijective for every $g \in G)$.

In Section 1 we show that kaleidoscopical configurations are tightly connected with classical combinatorial theme Transversality and, in the case $X=G$ and (left) regular action of $G$ on $G$, with factorization problem, well known in Factorization Theory of Groups, see [12], [13].

In Section 2 we introduce and describe the kaleidoscopical configurations (called splittable) which arise from the chains of $G$-invariant equivalences (imprimitivities) on $X$. If a $G$-space $X$ is primitive (the only $G$-invariant equivalences on $X$ are $X \times X$ and $\Delta_{X}$ ) then the splittable configurations in $X$ are only $X$ and the singletons.

In Section 3 we prove that every kaleidoscopical configuration in an isometrically homogeneous metric space with finite distance scale is splittable. For $n \geqslant 2$, we construct a plenty of kaleidoscopical configurations of cardinality $\mathfrak{c}$ in $\mathbb{R}^{n}$. These configurations are non-splittable because $\mathbb{R}^{n}$ is isometrically primitive. We don't know whether there exists a finite non-singleton or countable kaleidoscopical configurations in $\mathbb{R}^{n}, n \geqslant 2$.

In Section 4 we study the problem of splittability of kaleidoscopical configurations in finite Abelian groups and reformulate this problem in terms of the semi-Hajós property, which is a weak version of the Hajós property well-known in the factorization theory of groups [12], [13].

We note also that kaleidoscopical configurations in a sense are antipodal to monochromatizable configurations defined and studied in [4, Chapter 8]: a subset $A$ of a $G$ space $X$ is called monochromatizable if, for any finite coloring of $X$, there exists $g \in G$ such that $g A$ is monochrome. 


\section{Transversality and factorization}

Let $(X, \mathfrak{F})$ be a hypergraph. A subset $T \subseteq X$ is called an $\mathfrak{F}$-transversal if $|F \bigcap T|=1$ for each $F \in \mathfrak{F}$.

Proposition 1.1. A hypergraph $(X, \mathfrak{F})$ is kaleidoscopical if and only if $X$ can be partitioned into $\mathfrak{F}$-transversals.

Proof. For a kaleidoscopic hypergraph $(X, \mathfrak{F})$, let $\chi: X \rightarrow \kappa$ be a kaleidoscopical coloring. Then $X=\bigsqcup_{\alpha<\kappa} \chi^{-1}(\alpha)$ is a partition of $X$ into $\mathfrak{F}$-transversal.

On the other hand, if $X=\bigsqcup_{\alpha<\kappa} T_{\alpha}$ is a partition of $X$ into $\mathfrak{F}$-transversal subsets, then the coloring $\chi: X \rightarrow \kappa$ defined as $\chi(x)=\alpha \Leftrightarrow x \in T_{\alpha}$ is kaleidoscopical.

Let $X$ be a $G$-space, $A$ be a kaleidoscopical configuration in $X$. If $T$ is $G[A]$-transversal then $A$ is $G[T]$-transversal and $g T$ is $G[A]$ transversal for each $g \in G$.

We say that a kaleidoscopical configuration $A$ in $X$ is homogeneous if there exist a $G[A]$-transversal $T$ and a subset $H \subseteq X$ such that $X=\bigsqcup_{h \in H} h T$.

A subset $A$ of a group $G$ is defined to be complemented in $G$ if there exists a subset $B \subseteq G$ such that the multiplication mapping $\mu: A \times B \rightarrow G,(a, b) \mapsto a b$, is bijective. Following [13], we call the set $B$ a complementer factor to $A$, and say that $G=A B$ is a factorization of $G$. In this case, we have the partitions

$$
G=\bigsqcup_{a \in A} a B=\bigsqcup_{b \in B} A b .
$$

A subset $A \subseteq G$ is called doubly complemented if there are factorization $G=A B=B C$ for some subsets $B, C$ of $G$.

Proposition 1.2. For two subsets $A, B$ of a group $G$ the following conditions are equivalent:

1. $B$ is $G[A]$-transversal;

2. $G=A B^{-1}$ is a factorization of $G$.

Proof. (1) $\Rightarrow(2)$ For each $g \in G, g^{-1} A \cap B \neq \emptyset$, so $g \in A B^{-1}$. If $g=a_{1} b_{1}^{-1}=a_{2} b_{2}^{-1}$ for some $a_{1}, a_{2} \in A, b_{1}, b_{2} \in B$, then $g^{-1} a_{1}=b_{1}$ and $g^{-1} a_{2}^{-1}=b_{2}$ and by (1), $b_{1}=b_{2}$ and $a_{1}=a_{2}$, witnessing that $G=A B^{-1}$ is a factorization of $G$.

$(2) \Rightarrow(1)$ Fix any $g \in G$. The inclusion $g^{-1} \in A B^{-1}$ implies $g A \cap B \neq \emptyset$. If $g a_{1}=b_{1}$ and $g a_{2}=b_{2}$ for some $a_{1}, a_{2} \in A, b_{1}, b_{2} \in B$, then $g^{-1}=a_{1} b_{1}^{-1}=a_{2} b_{2}^{-1}$ and by (2), $b_{1}=b_{2}$, witnessing that $|g A \cap B|=1$.

Corollary 1.3. Each kaleidoscopical configuration in a group $G$ is complemented.

Proof. Given a kaleidoscopical configuration $A \subset G$, fix an $A$-kaleidoscopical coloring $\chi: G \rightarrow C$. We choose a color $c \in C$, consider the monochrome class $B=\chi^{-1}(b) \subset G$ and observe that for every $g \in G|g A \cap B|=1$ by the definition of $A$-kaleidoscopical coloring. By Proposition 1.2, $G=A B^{-1}$ is a factorization, so $A$ is complemented in $G$. 
Proposition 1.4. A subset $A$ of a group $G$ is doubly complemented if and only if $A$ is a homogeneous kaleidoscopical configuration.

Proof. Let $G=A B=B C$ be factorizations of $G$. By Proposition $1.2, B^{-1}$ is a $G[A]$ transversal. Since $G=\bigsqcup_{c \in C} c^{-1} B^{-1}$, we conclude that $A$ is a homogeneous kaleidoscopical configuration.

Let $A$ be a homogeneous kaleidoscopical configuration. We choose a $G[A]$-transversal $T$ and a subset $H \subseteq G$ such that $G=\bigsqcup_{h \in H} h T$. By proposition $1.2, G=A T^{-1}$. Since $G=\bigsqcup_{h \in H} h T, G=T^{-1} H^{-1}$ is a factorization. Hence, $A$ is doubly complemented.

Corollary 1.5. For a subset $A$ of an Abelian group $G$, the following statements are equivalent:

1. A is complemented;

2. A is a kaleidoscopical configuration;

3. A is a homogeneous kaleidoscopical configuration.

Question 1.6. Is each complemented subset of a (finite) group kaleidoscopical?

Proposition 1.7. Let $X$ be a transitive $G$-space, $x \in X, G_{x}=\{g \in G: g x=x\}$, $\gamma_{x}: G \rightarrow X, \gamma_{x}(g)=g x, s: X \rightarrow G$ be a section of $\gamma_{x}$. Let $A$ be a subset of $X, T$ be $a$ $G[A]$-transversal. Then

1. $s(T)$ is a $G\left[\gamma_{x}^{-1}(A)\right]$-transversal;

2. $|G|=\left|G_{x}\right||A||T|$.

Proof. The statement (1) is evident. The statement (2) follows from (1) and Proposition 1.2 .

Corollary 1.8. Let $A$ be a kaleidoscopical configuration in a finite transitive $G$-space $X$ with a kaleidoscopical coloring $\chi: G \rightarrow k$. Then $\chi^{-1}(0)=\cdots=\chi^{-1}(k-1)$ and $|X|=|A|\left|\chi^{-1}(0)\right|$.

Proof. We may suppose that $G$ is a subgroup of the group of all permutations of $X$ so $G$ is finite. Since $|G|=|X|\left|G_{x}\right|$, we can apply Proposition 1.7(2).

Proposition 1.9. Let $\kappa$ be an infinite cardinal, $(X, \mathfrak{F})$ be a hypergraph such that $|\mathfrak{F}|=\kappa$ and $|F|=\kappa$ for each $F \in \mathfrak{F}$. If $\left|F \cap F^{\prime}\right|<c f \kappa$ for all distinct $F, F^{\prime} \in \mathfrak{F}$ then there is a disjoint family $\mathfrak{T}$ of $\mathfrak{F}$-transversals such that $|\mathfrak{T}|=\kappa$ and $|T|=\kappa$ for each $T \in \mathfrak{T}$

Proof. We enumerate $\mathfrak{F}=\left\{F_{\alpha}: \alpha<\kappa\right\}$ and choose inductively the subsets $\left\{V_{\alpha} \subset F_{\alpha}\right.$ : $\alpha<\kappa\}$ such that the family $\left\{F_{\alpha} \backslash V_{\alpha}: \alpha<\kappa\right\}$ is disjoint and $\left|F_{\alpha} \backslash V_{\alpha}\right|=\kappa$ for each $\alpha<\kappa$. Let $F_{\alpha} \backslash V_{\alpha}=\left\{t_{\alpha \beta}: \beta<\kappa\right\}, T_{\beta}=\left\{t_{\alpha \beta}: \alpha<\kappa\right\}$. Then $\mathfrak{T}=\left\{T_{\beta}: \beta<\kappa\right\}$ is the desired family. 
For a hypergraph $(X, \mathfrak{F}), x \in X$ and $A \subseteq X$, we put

$$
\begin{gathered}
S t(x, \mathfrak{F})=\bigcup\{F \in \mathfrak{F}: x \in F\}, \\
S t(A, \mathfrak{F})=\bigcup\{S t(a, F): a \in A\} .
\end{gathered}
$$

Proposition 1.10. A hypergraph $(X, \mathfrak{F})$ is kaleidoscopical provided that, for some infinite cardinal $\kappa$, the following two conditions are satisfied:

1. $|\mathfrak{F}| \leqslant \kappa$ and $|F|=\kappa$ for each $F \in \mathfrak{F}$;

2. for any subfamily $\mathfrak{A} \subset \mathfrak{F}$ of cardinality $|\mathfrak{A}|<\kappa$ and any subset $B \subset X \backslash(\bigcup \mathfrak{A})$ of cardinality $|B|<\kappa$ the intersection $\operatorname{St}(B, \mathfrak{F}) \cap(\bigcup \mathfrak{A})$ has cardinality less than $\kappa$.

Proof. Let $\lambda=|\mathfrak{F}|$ and $\mathfrak{F}=\left\{F_{\alpha}: \alpha<\lambda\right\}$ be an injective enumeration of $\mathfrak{F}$. By induction we shall construct a transfinite sequence $\left(\chi_{\alpha}: F_{\alpha} \rightarrow \kappa\right)_{\alpha<\lambda}$ of bijective colorings such that for any ordinals $\alpha<\beta<\lambda$

$\left(1_{\alpha \beta}\right)$ the colorings $\chi_{\alpha}$ and $\chi_{\beta}$ coincide on $F_{\alpha} \cap F_{\beta}$;

$\left(2_{\alpha \beta}\right)$ no distinct points $a \in F_{\alpha}$ and $b \in F_{\beta}$ with $\chi_{\alpha}(a)=\chi_{\beta}(b)$ lie in some hyperedge $F \in \mathfrak{F}$.

Assume that for some ordinal $\gamma<\lambda$ we have constructed a sequence of colorings $\left(\chi_{\alpha}\right)_{\alpha<\gamma}$ satisfying the conditions $\left(1_{\alpha \beta}\right)$ and $\left(2_{\alpha \beta}\right)$ for all $\alpha<\beta<\gamma$.

Let us define a bijective coloring $\chi_{\gamma}: F_{\gamma} \rightarrow \kappa$. First we show that the union

$$
F_{\gamma}^{\prime}=\bigcup_{\alpha<\gamma} F_{\gamma} \cap F_{\alpha}
$$

has cardinality $\left|F_{\gamma}^{\prime}\right|<\kappa$. Observe that for each $\alpha<\gamma$ we get $F_{\alpha} \not \subset F_{\gamma}$. Assuming conversely that $F_{\alpha} \subsetneq F_{\gamma}$ and taking any point $v \in F_{\gamma} \backslash F_{\alpha}$ we conclude that the intersection $F_{\alpha} \cap \mathcal{S} t(v, \mathfrak{F}) \supset F_{\alpha} \cap F_{\gamma}=F_{\alpha}$ has cardinality $\geqslant \kappa$, which contradicts the condition (2) of the theorem.

Therefore, for each $\alpha<\gamma$ we can choose a point $v_{\alpha} \in F_{\alpha} \backslash F_{\gamma}$. Then for the set $B=\left\{v_{\alpha}: \alpha<\gamma\right\}$ the set $F_{\gamma}^{\prime} \subset F_{\gamma} \cap \mathcal{S t}(B, \mathfrak{F})$ has cardinality $\left|F_{\gamma}^{\prime}\right| \leqslant\left|F_{\gamma} \cap \mathcal{S t}(A, \mathfrak{F})\right|<\kappa$ according to (2).

For every point $x \in F_{\gamma} \backslash F_{\gamma}^{\prime}$ and every ordinal $\alpha<\gamma$, we consider the sets $\mathcal{S} t(x, \mathfrak{F}) \cap F_{\alpha}$ and $C_{\alpha}(x)=\chi_{\alpha}\left(\mathcal{S} t(x, \mathfrak{F}) \cap F_{\alpha}\right) \subset \kappa$. The condition (2) implies that the set $C(x)=$ $\bigcup_{\alpha<\gamma} C_{\alpha}(x)$ has cardinality $|C(x)|<\kappa$.

Let $\prec$ be any well-ordering on the set $F_{\gamma}$ such that $F_{\gamma}^{\prime}$ coincides the initial segment $\left\{x \in F_{\gamma}: x<y\right\}$ for some point $y \in F_{\gamma}$. Consider the coloring $\chi_{\gamma}: F_{\gamma} \rightarrow \kappa$ defined by $\chi_{\gamma}(x)=\chi_{\alpha}(x)$ if $x \in F_{\gamma} \cap F_{\alpha}$ for some $\alpha<\gamma$ and

$$
\chi_{\gamma}(x)=\min \{\kappa \backslash(C(x) \cup\{\chi(y): y \prec x\})\}
$$

if $x \in F_{\gamma} \backslash F_{\gamma}^{\prime}$. 
Let us show that the coloring $\chi_{\gamma}: F_{\gamma} \rightarrow \kappa$ is bijective. The injectivity of $\chi_{\gamma}$ follows from the definition of $\chi_{\gamma}$ and the conditions $\left(2_{\alpha \beta}\right), \alpha<\beta<\gamma$.

The surjectivity of $\chi_{\gamma}$ will follow as soon as we check that for each color $c \in \kappa \backslash \chi_{\gamma}\left(F_{\gamma}^{\prime}\right)$ the set $F_{\gamma}(c)=\left\{x \in F_{\gamma} \backslash F_{\gamma}^{\prime}: c \in C(x)\right\}$ has cardinality $<\kappa$. Observe that $c \in C(x)$ if and only if there is $\alpha<\gamma$ and a point $a \in F_{\alpha} \backslash F_{\gamma}$ such that $\chi_{\alpha}(a)=c$ and $x \in \mathcal{S t}(a, \mathfrak{F})$. The set $A_{c}=\bigcup_{\alpha<\gamma} \chi_{\alpha}^{-1}(c) \backslash F_{\gamma}$ has size $\left|A_{c}\right| \leqslant \gamma<\kappa$, and by the condition (2), the set $F_{\gamma}(c) \subset F_{\gamma} \cap \mathcal{S t}\left(A_{c}, \mathfrak{F}\right)$ has cardinality $<\kappa$. This completes the proof of the bijectivity of the coloring $\chi_{\gamma}$.

The conditions $\left(1_{\alpha \gamma}\right)$ and $\left(2_{\alpha, \gamma}\right)$ for all $\alpha<\gamma$ follow from the definition of the coloring $\chi_{\gamma}$. This completes the inductive step of the construction of the sequence $\left(\chi_{\alpha}\right)_{\alpha<\lambda}$.

After completing the inductive construction, let $\chi: V \rightarrow \kappa$ be any coloring such that $\left.\chi\right|_{F_{\alpha}}=\chi_{\alpha}$ for all $\alpha<\lambda$. The conditions $\left(1_{\alpha \beta}\right)$ guarantee that the coloring $\chi$ is welldefined. The bijectivity of the colorings $\chi_{\alpha}, \alpha<\lambda$, ensures the kaleidoscopicity of the coloring $\chi$.

We conclude this section with short discussion of possibilities of transfering above notions and results to quasigroups.

We recall that a quasigroup is a set $X$ endowed with a binary operation $*: X \times X \rightarrow X$ such that, for every $a, b \in X$, the system of equations $a * x=b, y * a=b$ has a unique solution $x=a \backslash b, y=b / a$ in $X$.

In an obvious way the notion of a kaleidoscopical configuration generalizes to quasigroup.

A subset $A$ of a quasigroup $X$ is called

- kaleidoscopical if there is a coloring $\chi: X \rightarrow C$ such that $\left.\chi\right|_{x * A}: x * A \rightarrow C$ is bijective for all $x \in X$;

- complemented if there is a subset $B \subset X$ such that the right division $\delta: B \times A \rightarrow X$, $\delta(b, a)=b / a$ is bijective;

- doubly complemented if there exists a complemented subset $B \subset X$ such that the multiplication $\mu: A \times B \rightarrow X, \mu(a, b)=a * b$, is bijective;

- self-complemented if the maps $\mu: A \times A \rightarrow X, \mu(x, y)=x * y$, and $\delta: A \times A \rightarrow X$, $\delta(x, y)=x / y$, are bijective.

It follows from the proof of Proposition 1.2 that each kaleidoscopical subset in a quasigroup is complemented. In contrast, Proposition 1.4 does not generalize to quasigroup.

Example 1.11. There exists a quasigroup $X$ of order $|X|=9$ that contains a selfcomplemented subset $A \subset X$, which is not kaleidoscopical.

Proof. It is well-known that finite quasigroups can be identified with Latin squares, i.e., $n \times n$ matrices whose rows and columns are permutations of the set $\{1, \ldots, n\}$. For $r, s \leqslant n$ an $(r \times s)$-matrix $\left(x_{i j}\right)$ is called a partial Latin $(r \times s)$-rectangle if $x_{i j} \in\{1,2, \ldots, n\}$ and $x_{l j} \neq x_{i j} \neq x_{i k}$ for any $1 \leqslant i \neq l \leqslant r$ and $1 \leqslant j \neq k \leqslant s$. By the result of Ryser [7] 
(see also Lemma 1 in [1, p.214]) each partial latin $(r \times s)$-rectangle can be completed to a Latin $(n \times n)$-square if and only if each number $i \in\{1, \ldots, n\}$ appears in the rectangle not less than $r+s-n$ times. This extension result allows us to find a quasigroups operation on $X=\{1, \ldots, 9\}$ whose multiplication table has the following first three columns:

\begin{tabular}{c|ccc}
$*$ & 1 & 2 & 3 \\
\hline 1 & 1 & 4 & 5 \\
2 & 6 & 2 & 7 \\
3 & 8 & 9 & 3 \\
\hline 4 & 4 & 1 & 6 \\
5 & 5 & 6 & 1 \\
\hline 6 & 2 & 7 & 8 \\
7 & 7 & 8 & 2 \\
8 & 3 & 5 & 9 \\
9 & 9 & 3 & 4 \\
\hline
\end{tabular}

Looking at this table we can see that the set $A=\{1,2,3\}$ is self-complemented as $A * A=X=A / A$. Assuming that $A$ is kaleidoscopical, find a coloring $\chi: X \rightarrow A$ such that $\left.\chi\right|_{x * A}$ is bijective for each $x \in X$. Since $1 * A=\{1,4,5\}$ and $4 * A=\{4,1,6\}$, the elements 5 and 6 have the same color, which is not possible as $5 * A=\{5,6,1\}$ and $\left.\chi\right|_{5 * A}$ is bijective.

\section{$2 \quad$ Splitting}

In this section we present a simple construction of kaleidoscopical configurations in arbitrary $G$-space, called the splitting construction. Kaleidoscopic subsets constructed in this way will be called splittable.

First we recall some definitions. A mapping $\varphi: X \rightarrow Y$ between $G$-spaces is called equivariant if $\varphi(g x)=g \varphi(x)$ for all $g \in G$ and $x \in X$. It is easy to see that each equivariant mapping between transitive $G$-spaces is surjective and homogeneous.

A function $\varphi: X \rightarrow Y$ is defined to be homogeneous if it is $\kappa$-to- 1 for some non-zero cardinal $\kappa$. The latter means that $\left|\varphi^{-1}(y)\right|=\kappa$ for all $y \in Y$.

Proposition 2.1. Let $\kappa$ be a non-zero cardinal, $\pi: X \rightarrow Y$ be an $\kappa$-to-1 equivariant mapping between two $G$-spaces and $s: Y \rightarrow X$ be a section of $\varphi$. Let $K \subset Y$ be a kaleidoscopic subset and $\chi: Y \rightarrow C$ be an $K$-kaleidoscopical coloring. Then:

1. the preimage $\bar{K}=\pi^{-1}(K)$ is a kaleidoscopical configuration in $X$ with respect to any coloring $\bar{\chi}: X \rightarrow C \times \kappa$ such that for each $y \in Y$ the restriction $\left.\bar{\chi}\right|_{\varphi^{-1}(y)}$ : $\pi^{-1}(y) \rightarrow\{\chi(y)\} \times \kappa$ is bijective;

2. the image $\tilde{K}=s(K)$ is a kaleidoscopical configuration in $X$ with respect to the $\tilde{K}$-kaleidoscopical coloring $\tilde{\chi}=\chi \circ \pi: X \rightarrow C$. 
Proof. 1. Given any element $g \in G$, we need to check that the restriction $\left.\bar{\chi}\right|_{g} \bar{K}: g \bar{K} \rightarrow$ $C \times \kappa$ is bijective. To see that it is surjective, take any color $(c, \alpha) \in C \times \kappa$ and using the surjectivity of $\left.\chi\right|_{g K}: g K \rightarrow C$, find a point $y \in g K$ with $\chi(y)=c$. Since the restriction $\left.\bar{\chi}\right|_{\pi^{-1}(y)}: \pi^{-1}(y) \rightarrow\{c\} \times \kappa$ is bijective, there is a point $x \in \pi^{-1}(y) \subset \pi^{-1}(g K)=g \bar{K}$ with $\bar{\chi}(x)=(c, \alpha)$, so $\left.\bar{\chi}\right|_{g \bar{K}}$ is surjective.

To see that it is injective, take any two distinct points $x, x^{\prime} \in g \bar{K}$. If $\pi(x)=\pi\left(x^{\prime}\right)$, then for the point $y=\pi(x)=\pi\left(x^{\prime}\right) \in g \pi(K)=\pi(g K)$ the injectivity of the restriction $\left.\bar{\chi}\right|_{\pi^{-1}(y)}$ implies that $\bar{\chi}(x) \neq \bar{\chi}\left(x^{\prime}\right)$. If $\pi(x) \neq \pi\left(x^{\prime}\right)$, then the injectivity of $\left.\chi\right|_{g K}$ guarantees that $\chi(\pi(x)) \neq \chi\left(\pi\left(x^{\prime}\right)\right)$ and then $\bar{\chi}(x) \neq \bar{\chi}\left(x^{\prime}\right)$ as $\bar{\chi}(x) \in\{\chi(\pi(x))\} \times \kappa$ and $\bar{\chi}(x) \in$ $\left\{\chi\left(\pi\left(x^{\prime}\right)\right)\right\} \times \kappa$.

2. Given any element $g \in G$, we need to check that the restriction $\left.\tilde{\chi}\right|_{g \tilde{K}}: g \bar{K} \rightarrow C$ is bijective. To see that $\left.\tilde{\chi}\right|_{g \tilde{K}}$ is surjective, observe that

$$
\tilde{\chi}(g \tilde{K})=\chi \circ \pi(g \tilde{K})=\chi(g \pi(\tilde{K}))=\chi(g K)=C
$$

by the surjectivity of $\left.\chi\right|_{g K}: g K \rightarrow C$.

To see that $\left.\tilde{\chi}\right|_{g \tilde{K}}$ is injective, take any two distinct points $x, x^{\prime} \in \tilde{K}=s(K)$ and observe $\pi(x) \neq \pi\left(x^{\prime}\right)$. Since $\pi$ is equivariant, $\pi(g x)=g \pi(x) \neq g \pi\left(x^{\prime}\right)=\pi\left(g x^{\prime}\right)$. Since $\pi(g x), \pi\left(g x^{\prime}\right) \in g K$ and $\left.\chi\right|_{g K}$ is injective, $\tilde{\chi}(x)=\chi(\pi(g x)) \neq \chi\left(\pi\left(g x^{\prime}\right)\right)=\tilde{\chi}\left(\pi\left(g x^{\prime}\right)\right)$ are we are done.

Iterating the constructions from Proposition 2.1, we get the splitting construction of kaleidoscopical configurations.

Proposition 2.2. Let $X_{0} \rightarrow X_{1} \rightarrow \cdots \rightarrow X_{m}$ be a sequence of $G$-spaces linked by homogeneous $G$-equivariant mappings $\pi_{i}: X_{i} \rightarrow X_{i+1}, i<m$. Let $K_{i} \subset X_{i}, i \leqslant m$, be subsets such that for every $i<m$ either the restriction $\left.\pi_{i}\right|_{K_{i}}: K_{i} \rightarrow K_{i+1}$ is bijective or else $K_{i}=\pi_{i}^{-1}\left(K_{i+1}\right)$. If the set $K_{m}$ is kaleidoscopic in the $G$-space $X_{m}$, then for every $i \leqslant m$ the set $K_{i}$ is kaleidoscopic in the $G$-space $X_{i}$.

Proof. This proposition can be derived from Proposition 2.1 by the reverse induction on $i \in\{m, m-1, \ldots, 0\}$.

Proposition 2.2 can be alternatively written in terms of invariant equivalence relations.

Given an equivalence relation $E \subset X \times X$ on a set $X$ let $X / E=\left\{[x]_{E}: x \in X\right\}$ be the quotient space consisting of the equivalence classes $[x]_{E}=\{y \in X:(x, y) \in E\}, x \in X$. Denote by $q_{E}: X \rightarrow X / E, q_{E}: x \mapsto[x]_{E}$, the quotient mapping. For a subset $K \subset X$ let $K / E=\left\{[x]_{E}: x \in K\right\} \subset X / E$ and $[K]_{E}=\bigcup_{x \in K}[x]_{E} \subset X$.

Let $E$ be an equivalence relation on a set $X$. A subset $K \subset X$ is defined to be

- E-parallel if $K \cap[x]_{E}=[x]_{E}$ for all $x \in K$;

- E-orthogonal if $K \cap[x]_{E}=\{x\}$ for all $x \in K$.

Given two equivalence relations $E \subset F$ on $X$ we can generalize these two notions defining $K \subset X$ to be 
- $F / E$-parallel if $[K]_{E} \cap[x]_{F}=[x]_{F}$ for all $x \in K$;

- $F / E$-orthogonal if $[K]_{E} \cap[x]_{F}=[x]_{E}$ for all $x \in K$.

Observe that a set $K \subset X$ is $E$-parallel ( $E$-orthogonal) if and only if it is $E / \Delta_{X}$-parallel $\left(E / \Delta_{X}\right.$-orthogonal). Here $\Delta_{X}=\{(x, x): x \in X\}$ stands for the smallest equivalence relation on $X$.

An equivalence relation $E$ on a $G$-space $X$ is called $G$-invariant if for each $(x, y) \in E$ and any $g \in G$ we get $(g x, g y) \in E$. For a $G$-invariant equivalence relation $E$ on $X$ the quotient space $X / E$ is a $G$-space under the induced action

$$
G \times X / E \rightarrow X / E, \quad\left(g,[x]_{E}\right) \mapsto[g x]_{E}
$$

of the group $G$. In this case the quotient projection $q: X \rightarrow X / E$ is equivariant. $G$ Invariant equivalence relations on $G$-spaces are also called imprimitivities.

Proposition 2.3. Let $\Delta_{X}=E_{0} \subset E_{1} \subset \cdots \subset E_{m}$ be a sequence of $G$-invariant equivalence relations on a transitive $G$-space $X$. A subset $K \subset X$ is kaleidoscopic provided

1. the projection $K / E_{m}$ is kaleidoscopic in the $G$-space $X / E_{m}$;

2. for every $i<m$ the set $K$ is $E_{i+1} / E_{i}$-parallel or $E_{i+1} / E_{i}$-orthogonal.

Proof. For every $i \leqslant m$ consider the $G$-space $X_{i}=X / E_{i}$ and the subset $K_{i}=K / E_{i}$ in $X_{i}$. Since $E_{0}=\Delta_{X}$, the space $X_{0}$ coincides with $X$. Next, for every $i<m$, consider the equivariant mapping $\pi_{i}: X_{i} \rightarrow X_{i+1}, \pi_{i}:[x]_{E_{i}} \mapsto[x]_{E_{i+1}}$. This mapping is homogeneous because of the transitivity of the $G$-space $X_{i}$.

We claim that the mappings $\pi_{i}$ satisfy the requirements of Proposition 2.2. Indeed, if $K$ is $E_{i+1} / E_{i}$-parallel, then $K_{i}=\pi_{i}^{-1}\left(K_{i+1}\right)$. If $K$ is $E_{i+1} / E_{i}$-orthogonal, then the restriction $\left.\pi_{i}\right|_{K_{i}}: K_{i} \rightarrow K_{i+1}$ is bijective.

Now Proposition 2.2 implies that the set $K=K_{0}$ is kaleidoscopic in $X=X_{0}$.

Proposition 2.3 suggests the following notion that will be cenral in our subsequent discussion.

Definition 2.4. A (kaleidoscopic) subset $K$ in a $G$-space $X$ is called splittable if there is an increasing sequence of $G$-invariant equivalence relations

$$
\Delta_{X}=E_{0} \subset E_{1} \subset \cdots \subset E_{m}=X \times X
$$

such that for every $i<m$ the set $K$ is either $E_{i+1} / E_{i}$-parallel or $E_{i+1} / E_{i \text {-orthogonal. }}$

Proposition 2.3 implies that each splittable subset in a transitive $G$-space is kaleidoscopic. What about the inverse implication?

Problem 2.5. For which $G$-spaces $X$, every kaleidoscopical configuration $K \subset X$ is splittable? 


\section{Kaleidoscopical configurations in metric spaces}

Here we consider each metric space $(X, d)$ as a $G$-space endowed with the natural action of its isometry group $G=\operatorname{Iso}(X)$. If this action is transitive, then the metric space $X$ is called isometrically homogeneous.

Let us recall that a metric space $(X, d)$ is ultrametric if the metric $d$ satisfies the strong triangle inequality

$$
d(x, z) \leqslant \max \{d(x, y), d(y, z)\}
$$

for all $x, y, z \in X$. It follows that for every $\varepsilon \geqslant 0$ the relation

$$
E_{\varepsilon}=\left\{(x, y) \in X^{2}: d(x, y) \leqslant \varepsilon\right\} \subset X \times X
$$

is an invariant equivalence relation on $X$.

Theorem 3.1. Let $(X, d)$ be an isometrically homogeneous ultrametric space with the finite distance scale $d(X \times X)=\left\{\varepsilon_{0}, \varepsilon_{1}, \ldots, \varepsilon_{n}\right\}$ where $0=\varepsilon_{0}<\varepsilon_{1}<\ldots<\varepsilon_{n}$. Then every kaleidoscopical configuration $K$ in $X$ is $\left(E_{\varepsilon_{0}}, E_{\varepsilon_{1}}, \ldots, E_{\varepsilon_{n}}\right)$-splittable.

Proof. Assume conversely that $K$ is not $\left(E_{\varepsilon_{0}}, E_{\varepsilon_{1}}, \ldots, E_{\varepsilon_{n}}\right)$-splittable. Then for some $k<n$ the set $K$ is neither $E_{\varepsilon_{k+1}} / E_{\varepsilon_{k}}$-parallel nor $E_{\varepsilon_{k+1}} / E_{\varepsilon_{k}}$-orthogonal. We can assume that $k$ is the smallest number with that property. By $[x]_{\varepsilon_{i}}$ we shall denote the closed $\varepsilon_{i}$-ball $[x]_{E_{\varepsilon_{i}}}$ centered at a point $x \in X$.

Since $K$ is not $E_{\varepsilon_{k+1}} / E_{\varepsilon_{k}}$-orthogonal, there are two points $u, v \in K$ such that $\varepsilon_{k}<$ $d(u, v)=\varepsilon_{k+1}$. Since $K$ is not $E_{\varepsilon_{k+1}} / E_{\varepsilon_{k}}$-parallel, there are points $w \in K$ and $z \in X$ such that $\varepsilon_{k}<\inf _{x \in K} d(z, x)=d(z, w)=\varepsilon_{k+1}$.

Since $X$ is isometrically homogeneous, we can find an isometry $\varphi: X \rightarrow X$ such that $\varphi(w)=z$. Then $\varphi\left([w]_{\varepsilon_{k}}\right)=[z]_{\varepsilon_{k}}$ and we can define an isometry $\phi: X \rightarrow X$ letting

$$
\phi(x)= \begin{cases}\varphi(x) & \text { if } x \in[w]_{\varepsilon_{k}}, \\ \varphi^{-1}(x) & \text { if } x \in[z]_{\varepsilon_{k}}, \\ x & \text { otherwise. }\end{cases}
$$

The isometry $\phi$ swaps the balls $[w]_{\varepsilon_{k}}$ and $[z]_{\varepsilon_{k}}$ but does not move points outside the union $[w]_{\varepsilon_{k}} \cup[z]_{\varepsilon_{k}}$. Since $K$ is $\chi$-kaleidoscopic, the restrictions $\left.\chi\right|_{\phi(K)}$ and $\left.\chi\right|_{K}$ are bijections onto $C$. Consequently, $\chi(w)=\chi\left(z^{\prime}\right)$ for some point $z^{\prime} \in[z]_{\varepsilon_{k}}$. Taking into account that $d\left(w, z^{\prime}\right)=d(w, z)=\varepsilon_{k+1}=d(u, v)$ and $X$ is an isometrically homogeneous ultrametric space, we can construct an isometry $\psi: X \rightarrow X$ such that $\psi(u)=w$ and $\psi(v)=z^{\prime}$. For this isometry, $w, z^{\prime} \in \psi(K)$ and hence $\left.\chi\right|_{\psi(K)}$ is not injective, contradicting the choice of the coloring $\chi$.

Problem 3.2. Let $\{0,1\}^{\omega}$ be the Cantor space endowed with the standard ultrametric generating the product topology. Describe all kaleidoscopical configurations in $\{0,1\}^{\omega}$.

Remark 3.3. All closed kaleidoscopical configurations in $\{0,1\}^{\omega}$ can be characterized with usage of Theorem 3.1. Among them there are plenty of non-splittable configurations. 
A $G$-space $X$ is called primitive if each $G$-invariant equivalence relation on $X$ is either $\Delta_{X}$ or $X \times X$. Thus, each splittable configuration $K$ in a primitive $G$-space $X$ is trivial, i.e. either $K=X$ or $K$ is singleton. It is natural to ask whether every kaleidoscopical configuration in a primitive $G$-space is trivial?

The answer to this question is affirmative if $X$ is 2-transitive in the sense that for any pairs $(x, y),\left(x^{\prime}, y^{\prime}\right) \in X^{2} \backslash \Delta_{X}$ there is $g \in X$ such that $\left(x^{\prime}, y^{\prime}\right)=(g x, g y)$.

An example of a primitive $G$-space, which is not 2-transitive is the Euclidean space $\mathbb{R}^{n}$ of dimension $n \geqslant 2$ endowed with the action of its isometry group Iso $\left(\mathbb{R}^{n}\right)$. We show that $\mathbb{R}^{n}$ contain $2^{\mathfrak{c}}$ unsplittable kaleidoscopical configurations of cardinality $\mathfrak{c}$.

To construct a kaleidoscopic subset in $\mathbb{R}^{n}$, use Proposition 1.10 and the following auxiliary definition.

Let $(X, d)$ be a metric space. By $S(x, r)=\{y \in X: d(x, y)=r\}$ we denote the sphere of radius $r$ centered at a point $x \in X$.

Definition 3.4. A subset $K$ of a metric space $(X, d)$ is called rigid if for any distinct points $x, y, z \in K$ and numbers $r_{x}, r_{y}, r_{z} \in d(K \times K)$ the spheres $S\left(x, r_{x}\right), S\left(y, r_{y}\right), S\left(z, r_{z}\right)$ have no common point in $X \backslash K$.

Theorem 3.5. Let $X$ be a metric space and $G \subset \operatorname{Iso}(X)$ be a group of isometries of $X$. Each infinite rigit subset $K \subset X$ of cardinality $|K| \geqslant|G|$ is kaleidoscopical.

Proof. The kaleidoscopicity of the set $K$ will follow from Proposition 1.10 as soon as we check that the hypergraph $(V, \mathfrak{F})=(X,\{g K: g \in G\})$ satisfies the conditions (1)-(2) for the cardinal $\kappa=|K|$. Since $|G| \leqslant \kappa=|K|=|g K|$ for all $g \in G$, the condition (1) is satisfied.

To show that (2) holds, take any subset $A \subset G$ of cardinality $|A|<\kappa$ and any subset $B \in X \backslash A K$ of cardinality $|B|<\kappa$. We need to show that $|\mathcal{S t}(B, \mathfrak{F}) \cap A K|<\kappa$. This will follow from $\max \{|A|,|B|\}<\kappa$ as soon as we check that $|\mathcal{S} t(b, \mathfrak{F}) \cap a K| \leqslant 2$ for every $b \in B$ and $a \in A$. Assuming conversely that $\mathcal{S} t(b, \mathfrak{F}) \cap a K$ contains three pairwise distinct points $x, y, z$ we shall obtain a contradiction with rigidity of $K$ because $d(b, x), d(b, y), d(b, z) \in d(K \times K)$ and $b$ is the common point of the spheres $S(x, d(b, x))$, $S(y, d(b, y)), S(z, d(b, z))$.

To apply Theorem 3.5, we need an effective construction of rigid subsets in metric spaces.

Lemma 3.6. Any algebraically independent over $\mathbb{Q}$ subset $A$ of an affine line (identified with $\mathbb{R}$ ) in the Euclidean space $\mathbb{R}^{n}$ is rigid.

Proof. Let $a, b, c$ be pairwise distinct points of $A, b \in[a, c], x \in \mathbb{R}^{n} \backslash A$ and $d(x, a)=r_{a}$, $d(x, b)=r_{b}, d(x, c)=r_{c}$. Since $\cos (\angle a b x)=-\cos (\angle c b x)$, by the cosines theorem, we get

(*) $(c-b)(b-a)^{2}+(c-b) r_{b}^{2}-(c-b) r_{a}^{2}+(b-a)(c-b)^{2}+(b-a) r_{b}^{2}-(b-a) r_{c}^{2}=0$.

Assuming $r_{a}, r_{b}, r_{c} \in d(A, A)$ and taking into account that at most two of the three numbers $r_{a}, r_{b}, r_{c}$ can be equal, after corresponding substitutions and opening all brackets in $(*)$, we get a contradiction with algebraic independence of $A$. 
Now we are able to prove the promised:

Theorem 3.7. For $n>1$ the Euclidean space $\mathbb{R}^{n}$ contains $2^{\mathfrak{c}}$ many kaleidoscopic subsets. Proof. Apply Theorem 3.5 and Lemma 3.6.

Problem 3.8. Does the Euclidean space $\mathbb{R}^{n}$ of dimension $n \geqslant 2$ contain a non-trivial finite or countable kaleidoscopical subset?

If such a set $K$ exists, then its cardinality $|K|$ is not less that the chromatic number of $\mathbb{R}^{n}$.

We recall that the chromatic number $\chi(X)$ of a metric space $X$ is equal to the smallest number $\kappa$ of colors for which there is a coloring of $X$ without monochrome points at the distance 1 . It is known that $4 \leqslant \chi\left(\mathbb{R}^{2}\right) \leqslant 7$ but the exact value of $\chi\left(\mathbb{R}^{2}\right)$ is not known. There is a conjecture that $\chi\left(\mathbb{R}^{n}\right)=2^{n+1}-1$, see $[10, \S 47]$.

Problem 3.9. Is every finite kaleidoscopical configuration in a (finite) primitive G-space trivial?

Some examples of infinite $G$-spaces with only trivial finite kaleidoscopical configurations can be found in [4, Chapter 8]

A space $\mathbb{R}^{n}$ can also be considered as a $G$-space with respect to the group $G=$ $\operatorname{Aff}\left(\mathbb{R}^{n}\right)=\left\{\lambda x+a: \lambda \in \mathbb{R} \backslash\{0\}, a \in \mathbb{R}^{n}\right\}$ of all affine transformations. The kaleidoscopical configurations $K$ of cardinality $|K|<\mathfrak{c}$ in this space are singletons because any line that contains more then one point of a kaleidoscopical configuration has no distinct points of the same color. On the other hand, every affine subspace of $\mathbb{R}^{n}$ is kaleidoscopical. Moreover, using Proposition 1.10, we can construct $2^{\mathfrak{c}}$ non-splittable affine kaleidoscopical configuration of size $\mathfrak{c}$ in $\mathbb{R}^{n}$ for $n>1$.

Restricting ourself with only translations of $\mathbb{R}^{n}$, we get a kaleidoscopical configuration of any size $\kappa, 1 \leqslant \kappa \leqslant \mathfrak{c}$. It follows from well-known decomposition of $\mathbb{R}^{n}$ in the direct sum of rationals and the observation that $\mathbb{Z}$ has a kaleidoscopical configuration of any finite size.

\section{Hajós properties in groups and $G$-spaces}

In this section we reveal the relation of splittability of kaleidoscopical configurations in finite Abelian groups to the Hajós property introduced in [2] and studied in [8], [12], [13].

We recall that an Abelian group $G$ has the Hajós property if for each factorization $G=A B$ either $A$ or $B$ is periodic. A subset $A$ of a group $G$ is called periodic if $A=g A$ for some non-identity element $g \in G$. Finite Abelian groups with Hajós property were classified in [8]:

Theorem 4.1 (Hajós-Sands). A finite Abelian group $G$ has the Hajós property if and only if $G$ is isomorphic to a subgroup of a group that has one of the following types:

$\left(p^{n}, q\right),\left(p^{2}, q^{2}\right),\left(p^{2}, q, r\right),(p, q, r, s),(p, p),(p, 3,3),\left(3^{2}, 3\right)$,

$\left(p^{3}, 2,2\right),\left(p^{2}, 2,2,2\right),\left(p, 2^{2}, 2\right),(p, 2,2,2,2),(p, q, 2,2),\left(2^{n}, 2\right),\left(2^{2}, 2^{2}\right)$, where $p<q<r<s$ are distinct primes and $n \in \mathbb{N}$. 
A group $G$ is of type $\left(n_{1}, \ldots, n_{k}\right)$ if $G$ is isomorphic to the direct sum of cyclic groups $C_{n_{1}} \oplus \cdots \oplus C_{n_{k}}$.

Now let us define two weakenings of the Hajós property.

Definition 4.2. An Abelian group $G$ is defined to have

- the semi-Hajós property if each complemented subset $A \subsetneq G$ either is periodic or has a periodic complementer factor in $G$;

- the demi-Hajós property if for each factorization $G=A B$ one of the factors $A, B$ either is periodic or has a periodic complementer factor.

It is clear that for each Abelian group $G$

$$
\text { Hajós } \Rightarrow \text { semi-Hajós } \Rightarrow \text { demi-Hajós. }
$$

Problem 4.3. Is the semi-Hajós property of finite Abelian groups equivalent to the demiHajós property?

The demi-Hajós property was (implicitly) defined in [9] and follows from the quasiperiodicity of any factorization of the group. In contrast to the Hajós property, at the moment we have no classification of finite Abelian groups possessing the demi-Hajós property. It is even not known if each finite cyclic group has the demi-Hajós property, see Problem 5.4 in [13]. The best known positive result on the semi-Hajós property is the following version of Theorem 5.13 [13]:

Theorem 4.4 (Szabó, [11]). Each finite Abelian group $G$ of square-free order $|G|$ has the semi-Hajós property.

We say that a number $n$ is square-free if $n$ is not divisible by the square $p^{2}$ of any prime number $p$.

Surprisingly, the following problem of Fuchs and Sands [3, p.364], [9], [13, p.120] posed in 60 -ies still is open:

Problem 4.5. Has each finite Abelian group the demi-Hajós property?

The "semi" version of this problem also is open:

Problem 4.6. Has each finite Abelian group the semi-Hajós property?

The semi-Hajós property is tightly connected with the splittability of kaleidoscopical configurations. In order to state the precise result, let us generalize the definition of the semi-Hajós property to $G$-spaces.

Definition 4.7. A $G$-space $X$ has the semi-Hajós property if for each kaleidoscopical subset $K \subsetneq X$ there is a $G$-invariant equivalence relation $E \neq \Delta_{X}$ on $X$ such that $K$ is $E$-parallel or $E$-orthogonal and the set $K / E$ is kaleidoscopical in the $G$-space $X / E$. 
For finite Abelian groups this definition of the semi-Hajós property agrees with that given in Definition 4.2 .

Proposition 4.8. A finite Abelian group $G$ has the semi-Hajós property if and only if it has that property as a $G$-space.

Proof. Assume that the group $G$ has the semi-Hajós property. To show that the $G$-space $G$ has the semi-Hajós property, take any kaleidoscopical subset $A \subset G$. By Corollary 1.5, $A$ is complementable and hence has a complementer factor $B$. Since $G$ has the semi-Hajós property, either $A$ is periodic or else $A$ has a periodic complementer factor. In the latter case we can assume that the complementer factor $B$ is periodic. Consequently there is a non-trivial cyclic subgroup $H \subset G$ such that either $A+H=A$ or $B+H=B$. Consider the quotient group $G / H$ and the quotient homomorphism $q: G \rightarrow G / H$. By Lemma 2.6 of [13], the images $A / H=q(A)$ and $B / H=q(B)$ form a factorization $G / H=A / H \cdot B / H$ of the quotient group $G / H$. Consequently, the set $A / H$ is complemented in $G / H$ and by Corollary 1.5, it is kaleidoscopical in $G / H$.

The subgroup $H$ induces a $G$-invariant equivalence relation $E=\{(x, y) \in G: x-y \in$ $H\}$ whose quotient space $G / E$ coincides with the quotient group $G / H$. We claim that the set $A$ is either $E$-parallel or $E$-orthogonal. By the choice of the group $H$, we get $A=A+H$ or $B=B+H$. In the first case the set $A$ is $E$-parallel. In the second case $A$ is $E$-orthogonal as $(A-A) \cap H \subset(A-A) \cap(B-B)=\{0\}$.

Now assuming that the $G$-space $G$ has the semi-Hajós property, we shall prove that the group $G$ has the semi-Hajós property. Given any complemented subset $A \subset G$ we need to show that either $A$ is periodic or else $A$ has a periodic complementer factor. By Corollary 1.5, the set $A$ is kaleidoscopical in the $G$-space $G$. The semi-Hajós property of the $G$-space $G$ guarantees the existence of a $G$-invariant equivalence relation $E \neq \Delta_{G}$ on $G$ such that $A$ is $E$-parallel or $E$-orthogonal and $A / E$ is kaleidoscopical in $G / E$. It follows that the equivalence class $H=[0]_{E}$ of zero is a subgroup of the group $G$. Taking into account that $E$ is $G$-invariant, we conclude that $(x, y) \in E$ iff $x-y \in[0]_{E}$. So, $G / E$ coincides with the quotient group $G / H$. The set $A / H$, being kaleidoscopical, is complemented in $G / H$ according to Corollary 1.5. Consequently, there is a subset $B_{H} \subset G / H$ such that $G / H=A / H \cdot B_{H}$. Let $q: G \rightarrow G / H$ be the quotient mapping and $s: G / H \rightarrow G$ be any section of $q$.

Now consider two cases. If $A$ is $E$-parallel, then $A=A+H$ is periodic and complemented as $B=s\left(B_{H}\right)$ is a complementer factor to $A$ in $G$. If $A$ is $E$-orthogonal, then the complete preimage $B=q^{-1}\left(B_{H}\right)$ is a periodic complementer factor to $A$ in $G$.

Now we reveal the relation between the semi-Hajós property and the splittability of kaleidoscopical sets.

Proposition 4.9. If each kaleidoscopical subset of a transitive $G$-space $X$ is splittable, then $X$ has the semi-Hajós property.

Proof. To show that $X$ has the semi-Hajós property, fix any kaleidoscopical subset $K \subset X$. By our assumption, $K$ is $\left(E_{0}, \ldots, E_{m}\right)$-splittable by some increasing chain of invariant 
equivalence relations $\Delta_{X}=E_{0} \subset \cdots \subset E_{m}=X \times X$. For every $i \leqslant m$ consider the quotient $G$-space $X_{i}=X / E_{i}$ and let $q_{i}: X \rightarrow X_{i}$ be the quotient projection. Also let $K_{i}=q_{i}(K) \subset X_{i}$. By Proposition 2.2, $K_{i}$ is kaleidoscopical in the $G$-space $X_{i}$. In particular, $K_{1}$ is kaleidoscopical in $X_{1}=X / E_{1}$. By Definition 2.4, $K=K_{0}$ is either $E_{1}$-parallel or $E_{1}$-orthogonal. This means that $X$ has the semi-Hajós property.

Theorems 3.1 and Proposition 4.9 imply

Corollary 4.10. Each isometrically homogeneous ultrametric space with finite distance scale has the semi-Hajós property.

A $G$-space $Y$ is defined to be a quotient of a $G$-space $X$ if $Y$ is the image of $X$ under a $G$-equivariant mapping $f: X \rightarrow Y$.

Proposition 4.11. Each kaleidoscopical subset of a $G$-space $X$ is splittable provided that:

1. each quotient $G$-space of $X$ has the semi-Hajós property and

2. $X$ admits no strictly increasing infinite sequence $\left(E_{n}\right)_{n \in \omega}$ of $G$-invariant equivalence relations.

Proof. Assume that some kaleidoscopical subset $K \subset X$ is not splittable. Let $K_{0}=K$, $E_{0}=\Delta_{X}$, and $X_{0}=X / E_{0}=X$. Since $X$ has the semi-Hajós property, there is a $G$-invariant equivalence relation $E_{1} \neq \Delta_{X}$ on $X_{0}$ such that the set $K_{1}=K_{0} / E_{0}$ is kaleidoscopical in the $G$-space $X_{1}=X_{0} / E_{1}$ and $K_{0}$ is either $E_{1}$-parallel or $E_{1}$-orthogonal.

By our assumption, $K$ is not splittable, so $X_{1}$ is not a singleton. The $G$-space $X_{1}=X / E_{1}$, being a quotient of $X$, has the semi-Hajós property. Consequently, for the kaleidoscopical set $K_{1} \subset X_{1}$ there is a $G$-invariant equivalence relation $\tilde{E}_{2} \neq \Delta_{X_{1}}$ on $X_{1}$ such that the set $K_{1}$ is $\tilde{E}_{2}$-parallel or $\tilde{E}_{2}$-orthogonal and the quotient set $K_{2}=K_{1} / \tilde{E}_{1}$ is kaleidoscopical in the $G$-space $X_{2}=X_{1} / \tilde{E}_{1}$. Let $q_{2}^{1}: X_{1} \rightarrow X_{2}$ be the quotient projection. The composition $q_{2}^{1} \circ q_{1}: X \rightarrow X_{2}$ determines the $G$-invariant equivalence relation $E_{2}=\left\{\left(x, x^{\prime}\right) \in X^{2}: q_{2}^{1} \circ q_{1}(x)=q_{2}^{1} \circ q_{1}\left(x^{\prime}\right)\right\}$ on $X$ such that $X / E_{2}=X_{2}$ and $K_{2}=K / E_{2}$ and $K_{1}$ is either $E_{2} / E_{1}$-parallel or $E_{2} / E_{1}$-orthogonal.

Continuing by induction, we shall produce an infinite increasing sequence $\left(E_{n}\right)_{n \in \omega}$ of $G$-invariant equivalence relations on $X$ such that for every $n \in \mathbb{N}$ the set $K_{n}=K / E_{n}$ is kaleidoscopical in the $G$-space $X / E_{n}$ and $K$ is either $E_{n} / E_{n-1}$-parallel or $E_{n} / E_{n-1^{-}}$ orthogonal. But the existence of an infinite strictly increasing sequence of $G$-invariant equivalence relations on $X$ contradicts our assumption.

Since each quotient group of a finite Abelian group $G$ is isomorphic to a subgroup of $G$, Proposition 4.11 implies:

Corollary 4.12. If each subgroup of a finite Abelian group $G$ has the semi-Hajós property, then each kaleidoscopical subset $K \subset G$ is splittable.

Question 4.13. Assume that a finite Abelian group $G$ has the semi-Hajós property. Has each subgroup of $G$ that property? 
The classification of finite Abelian groups with Hajós property given in Theorem 4.1 implies that this property is inherited by subgroups. Because of that, Corollary 4.12 implies:

Corollary 4.14. For a finite Abelian group $G$ with the Hajós property, each kaleidoscopical subset $K \subset G$ is splittable.

Also Proposition 4.11 and Theorem 4.4 imply:

Corollary 4.15. For a finite Abelian group $G$ of square-free order $|G|$ each kaleidoscopical subset $K \subset G$ is splittable.

Remark 4.16. It follows from Proposition 4.9 and Corollary 4.12 that Question 4.13 and Problem 4.6 are equivalent (and both are open and apparently difficult).

According to an old result of Hajós [2], if in a factorization $\mathbb{Z}=A+B$ of the infinite cyclic group $\mathbb{Z}$ the factor $A$ is finite, then the factor $B$ is periodic. We do not know if the same is true for the groups $\mathbb{Z}^{n}$ with $n \geqslant 2$.

Acknowledgement. We thank referee for remarks, suggestions, and references.

\section{References}

[1] M. Aigner, G. Ziegler, Proofs from The Book, Springer-Verlag, Berlin, 2010.

[2] G. Hajós, Sur la factorisation des groupes abèliens, Časopis Pěst. Mat. Fys. 74 (1949), $157-162$.

[3] J.M. Irwin, E.A. Walker, Topics in Abelian Groups, Glenview, Illinois, 1963.

[4] I. Protasov, T. Banakh, Ball Structures and Colorings of Graphs and Groups, Math. Stud. Monogr. Ser, Vol. 11, VNTL Publisher, Lviv, 2003.

[5] I. Protasov, K. Protasova, Kaleidoscopical Graphs and Hamming codes, Voronoi's Impact on Modern Science, Book 4, Volume 1, Proc. 4th Intern. Conf. on Analytic Number Theory and Spatial Tesselations, Institute of Mathematics, NAS of Ukraine, Kyiv, 2008, 240-245.

[6] K.D. Protasova, Kaleidoscopical Graphs, Math. Stud., 18 (2002), 3-9.

[7] H.J. Ryser, A combinatorial theorem with an application to latin rectangles, Proc. Amer. Math. Soc. 2 (1951), 550-552.

[8] A.D. Sands, On the factorisation of finite Abelian groups II, Acta Math. Acad. Sci. Hungar. 13 (1962), 153-169.

[9] A.D. Sands, On a conjecture of G. Hajós, Glasgow Math. J. 15 (1974), 88-89.

[10] A. Soifer, The mathematical coloring book, Springer, 2009.

[11] S. Szabó, Groups admitting only quasi-periodic factorizations, Annales Sci. Budapest., Sect. Comp. 29 (2008), 239-243.

[12] S. Szabó, Topics in Factorization of Abelian Groups, Birkhäuser Basel, 2005.

[13] S. Szabó, A. Sands, Factoring groups into subsets, CRC Press, 2009. 